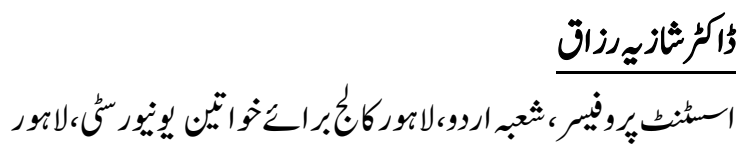

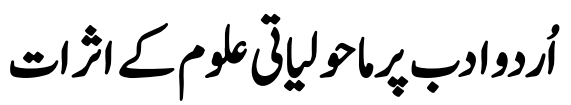

Dr Shazia Razzaq

Assistant Professor, Department of Urdu, Lahore College for Woemn University, Lahore.

\title{
Impact of Environmental Sciences on Urdu Literature
}

In view of the scientific studies of present age, Literature holds a unique status in scientific studies also a part of "ecology". My article is a study of the impact of different branches of ecological knowledge on Literature. My objective through this article is to highlight the exceptional characteristic and importance of literature and ecological studies as well as their relationship .The important fact which have revealed as a result of this research work is that literature and ecological knowledge are not aliens. The study shows that they are not inferior or superior to each other but are placed side by side just like members of one family or rays flowing from same source which is human being. Ecological knowledge is revolving around this source in a circle and the rays running there from absorbs in literature and so after getting fresh energy again becomes a part of circle revolving around the source. It is being discussed that literature and ecological knowledge are interlinked and are stepping ahead side by side.

Key Words: Literature, Scientific, Exceptional, Importance, Ecological, Inferior, Superior.

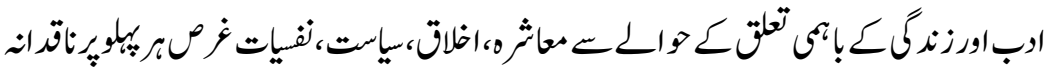

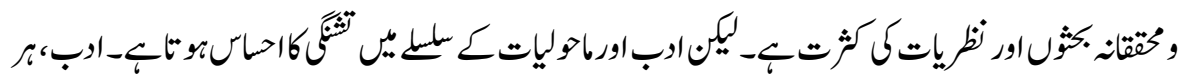

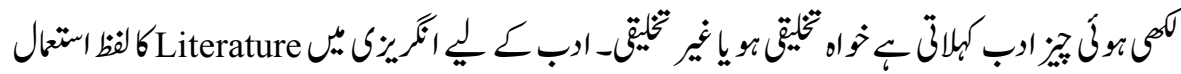

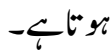


"Literature, a body of written works. The name has traditionally been applied to those imaginative works of poetry and prose distinguished by the intentions of their authors and the perceived aesthetic excellence of their execution". (1)

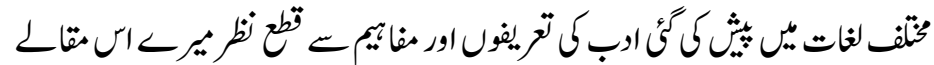

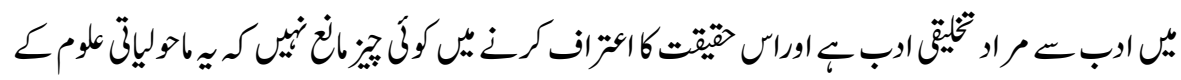

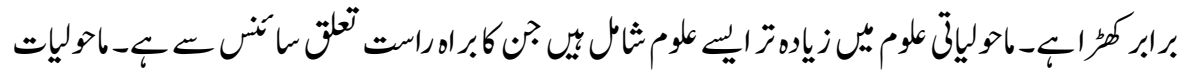

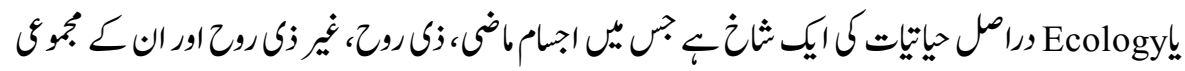

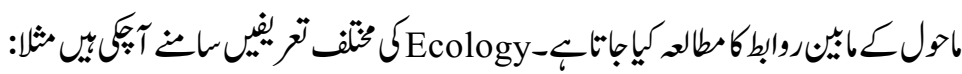

"The study of the inter relationship of organisms with their environment and eachother". (2)

"Ecology: is the science that studies the biata (living thing) the environment and their interactions. It comes from the Gree Oikos = House, Logos = Study .

Ecology is the study of ecosystems". (3)

$$
\text { :Essentials of Ecology }
$$

"The scientific study of the distribution and abundance of arganisms and the interactions that determine distribution and abundance". ${ }^{(4)}$

$$
\text { Concepts of Ecology” }
$$

"Hanns Reiter (1885) appears to have been the first to combine the words oikos (house) and logos (study of) to form the term ecology (Egerton 1977). There is consensus, however, that Haeckle gave definition and substance to the term, which he first used in 1866, in the following statement written in 1877:

By ecology we mean the body of knowledge concerning the economy of nature the investigation of the total relations of the animal both to its inorganic and to its organic environment, including above all, its friendly and inimical relation with those animals and 
plants with which it comes directly indirectly in to contract -in a word, ecology is the study of all the complex interrelations of referred to by Darwin as the conditions of the struggle for existence.

The science of ecology often inaccurately referred to as 'biology' in a narrow sense, has thus for formed the principal component what in commonly referred to as 'National History'. As is well shown by the numerous popular natural histories of both early and modern times, this subject has developed in the most closed relations with systematic zoology. The ecology of animals has been dealt with quite uncritically in natural history; but natural history has in any case hud the merit of keeping alive a widespread and interest in zoology". (5)

$$
\text { Ecology }
$$

"The French zoologist Isodone Geoffroyst. Hilaire had proposed the term of ecology for "The study of the relations of the organism within the family and society in the aggregate and in the community". ${ }^{(6)}$

"The English naturalists St. George Jackson Mivart coined the term bexicology, which he defined in 1894 as "Devoted to the study of the relations which exists between the organism and their environment as regards the nature of the locality they frequent, the temperatures and the amounts of light which suit them, and their relations to other organism as enemies, rivals, or accidental and involuntary benefactors". (7)

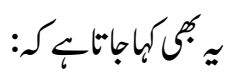

"As a scientific discipline, ecology does not dictate what is "right" or wrong but after understanding the principles, it is the humans should decide on how to manage the environment. To manage the environment, knowledge of ecology is essential". (8) 


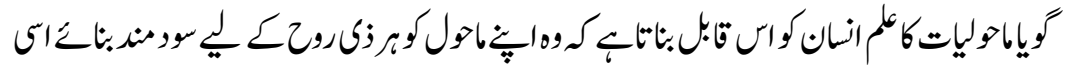

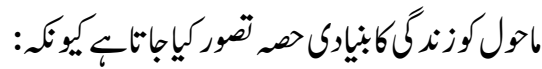

"All living things on this earth breath alike ."

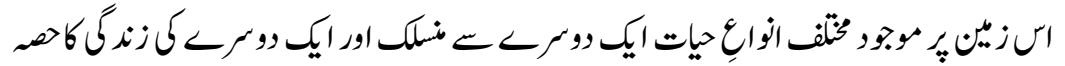

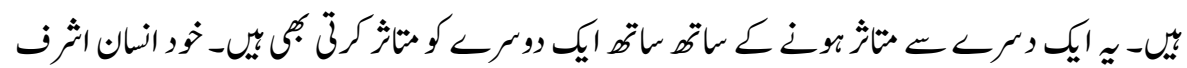

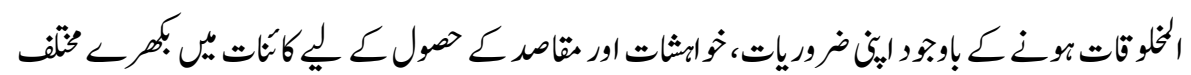

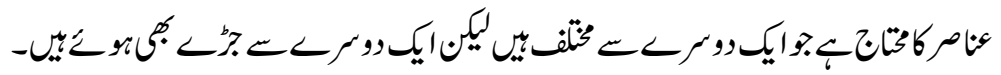

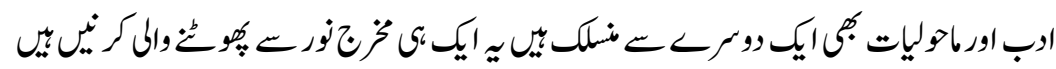

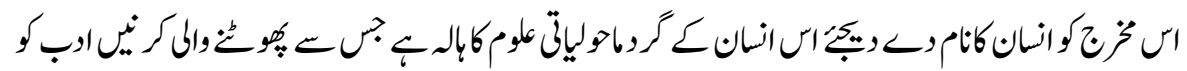

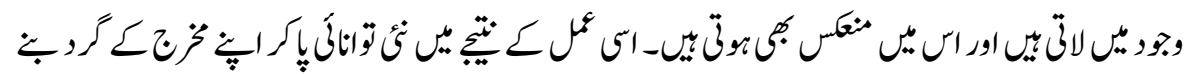

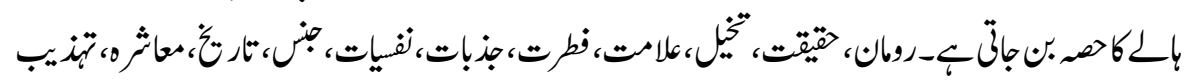

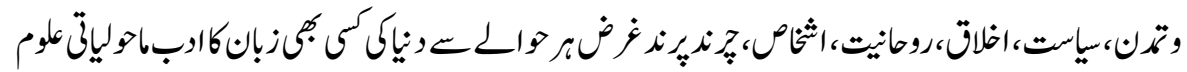

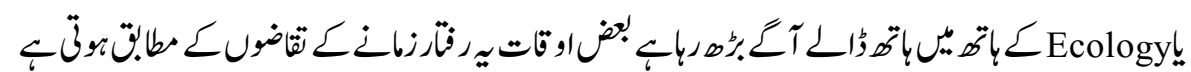

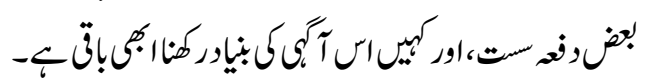

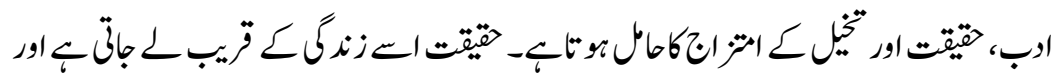

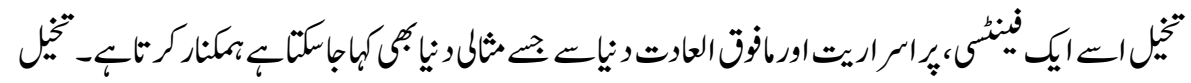

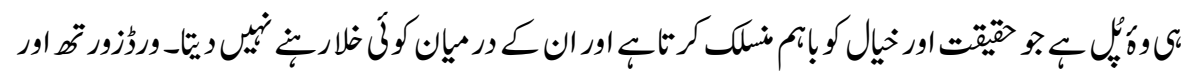

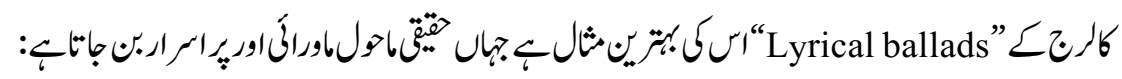

All in a hot and copper sky

The bloody sun at moon

Right up above the most did stand

No bigger than the moon

Day after day, day after day

We stuck, no breath no motion

As idle as a painted ship

upon a painted ocean

Water, Water, every where 
And all the boards did shrink

Water, Water, every where

No any drop to drink". ${ }^{(9)}$

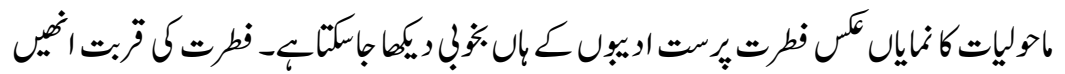

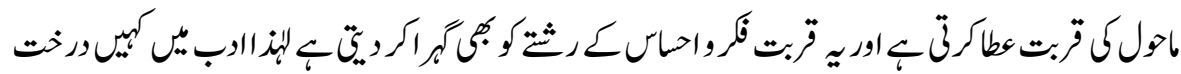

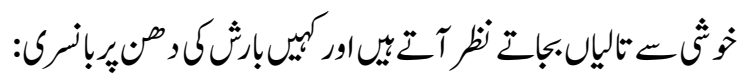

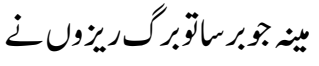

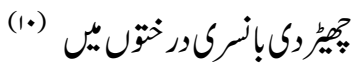

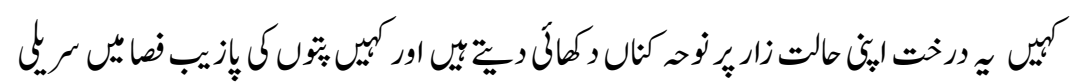

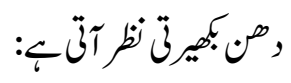

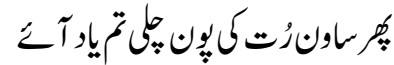

(11)

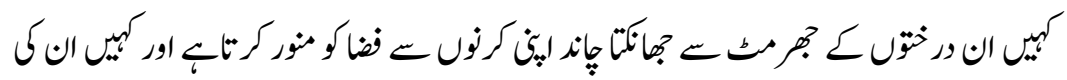

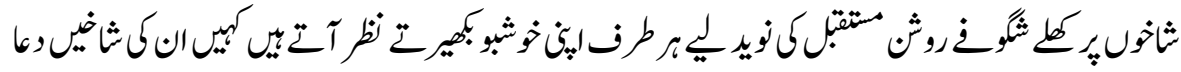

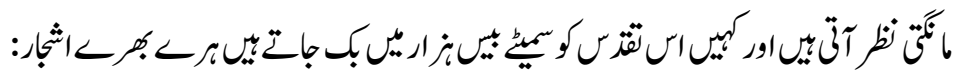

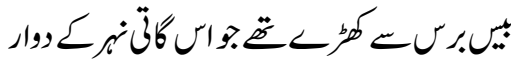

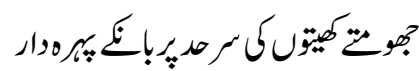

$$
\begin{aligned}
& \text { }
\end{aligned}
$$

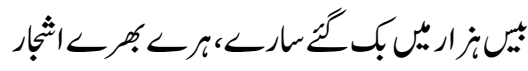

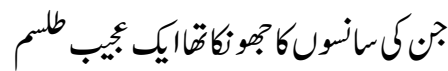

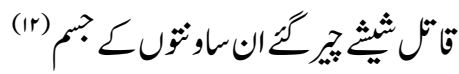




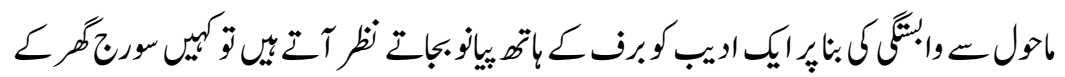

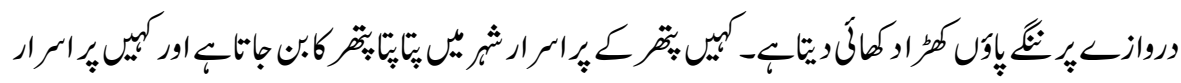

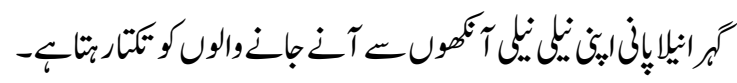

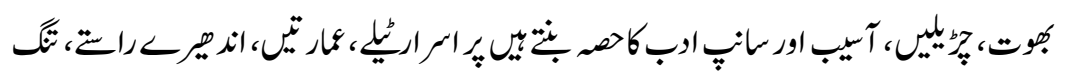

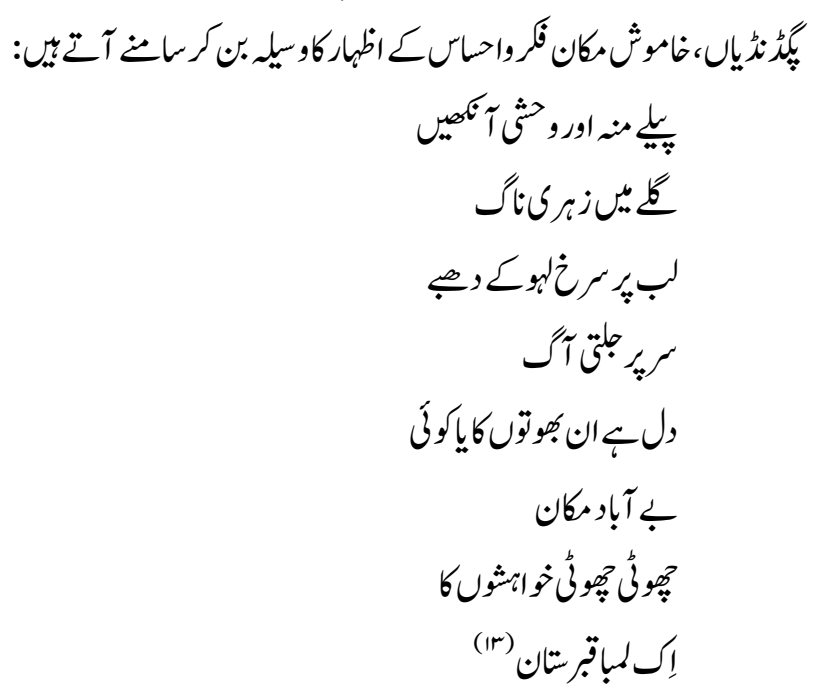

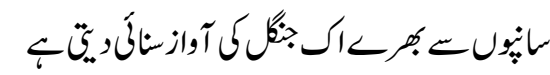

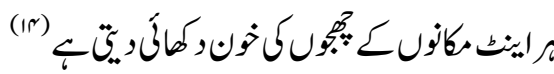

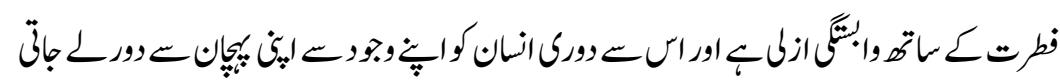

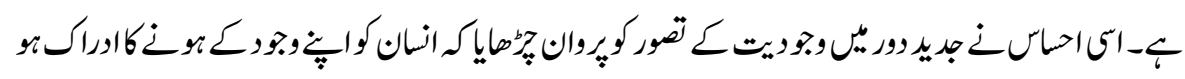

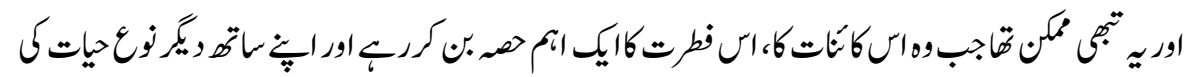

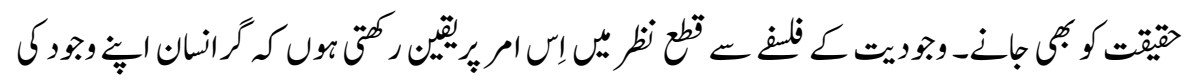

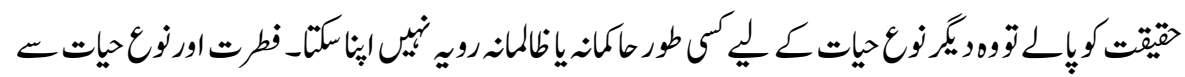

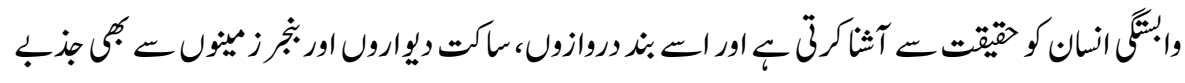

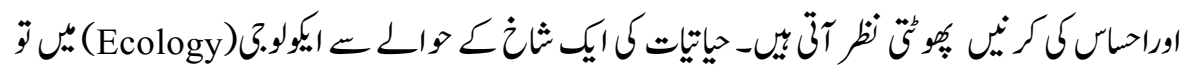

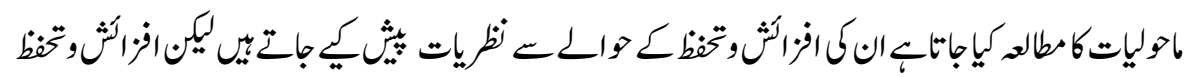




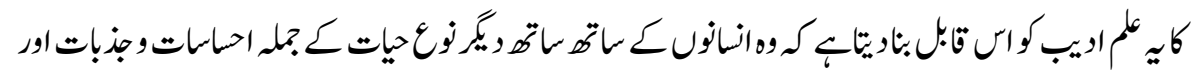

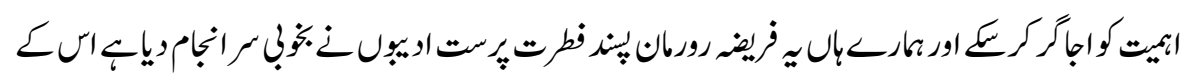

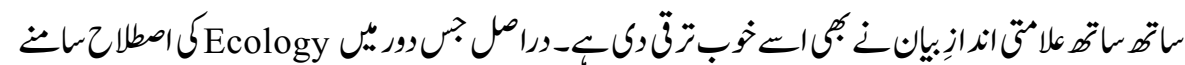

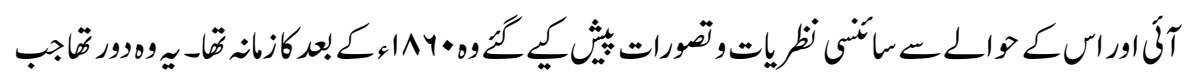

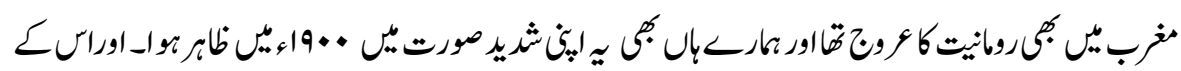

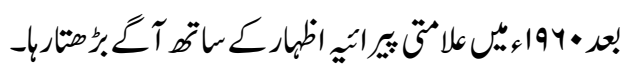

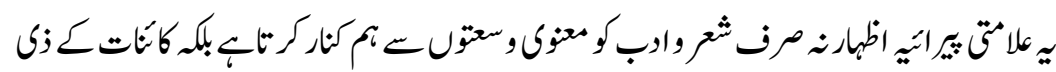

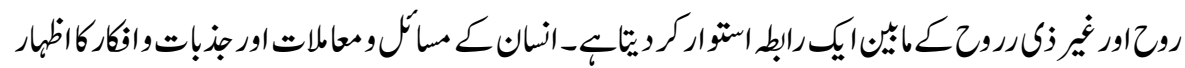

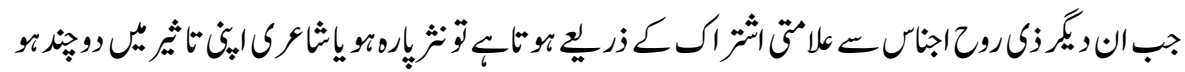

$$
\begin{aligned}
& \text { جإنب }
\end{aligned}
$$

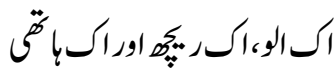

$$
\begin{aligned}
& \text { ثرأن }
\end{aligned}
$$

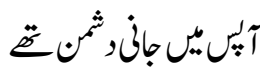

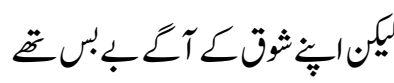

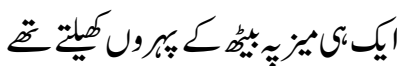

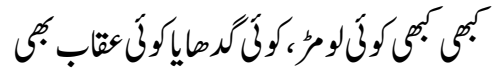

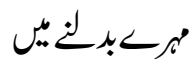

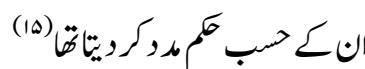

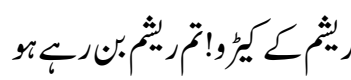

$$
\begin{aligned}
& \text { (14) }
\end{aligned}
$$

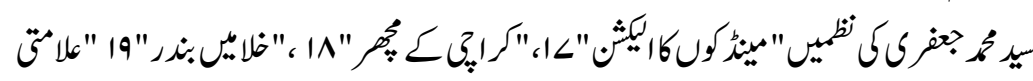

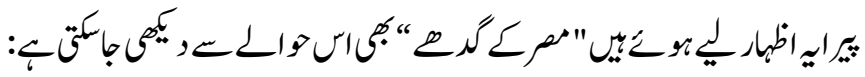




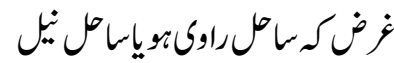

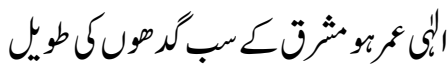

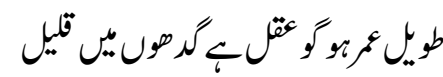

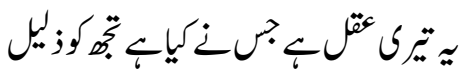

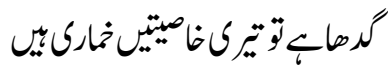

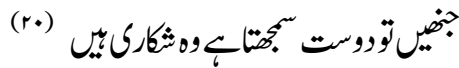

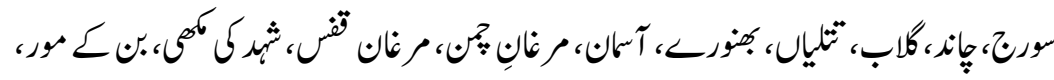

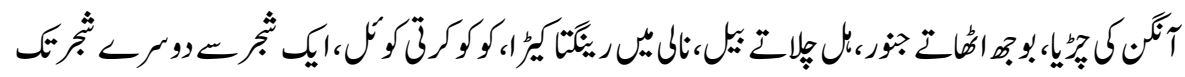

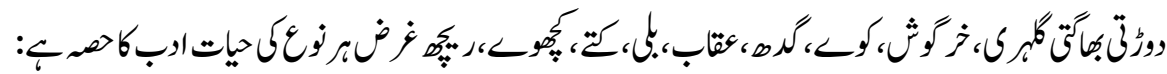

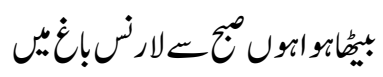

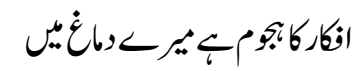

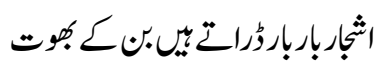

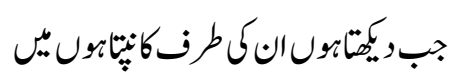

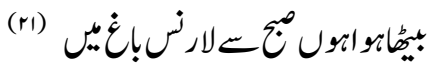

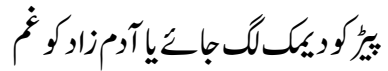

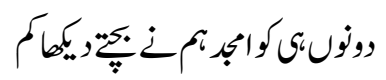

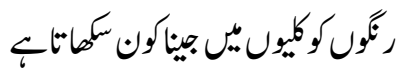

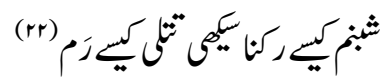

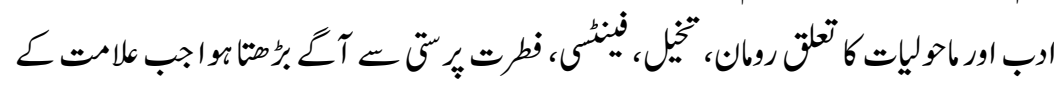

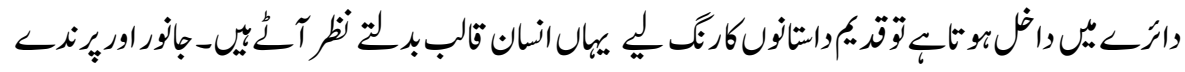

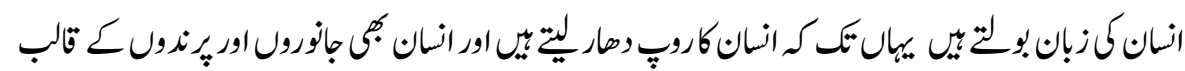

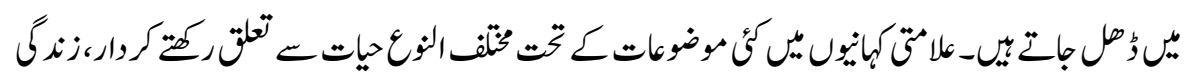

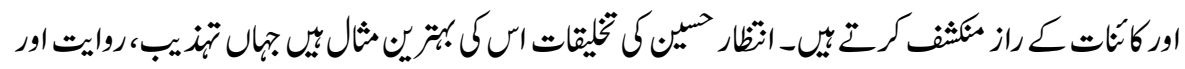




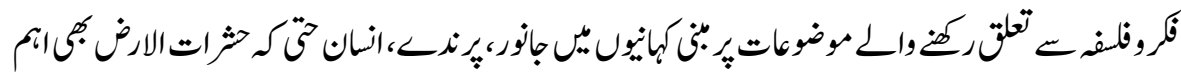

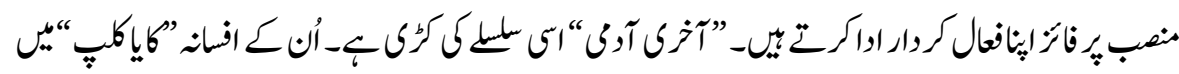

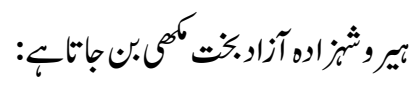

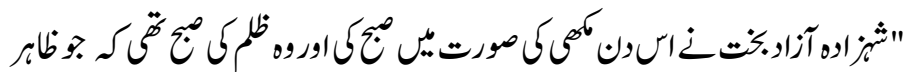

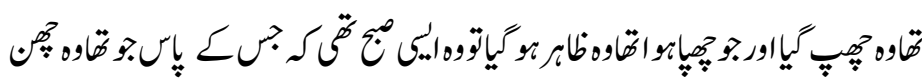

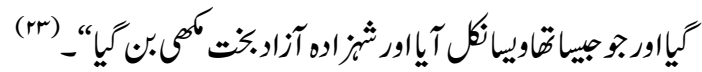
"ونويا-ن كياكوياكيإيا":

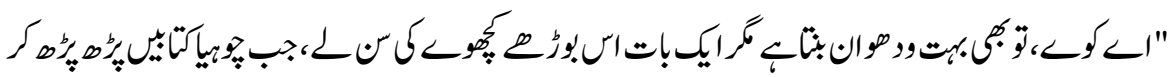

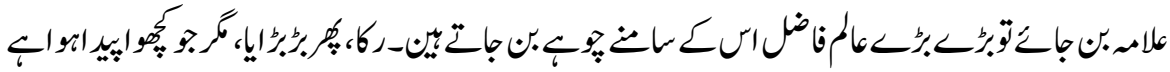

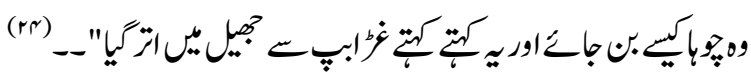

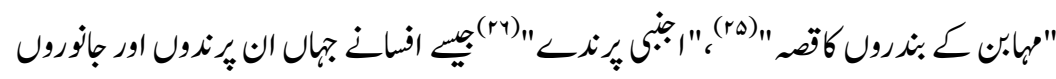

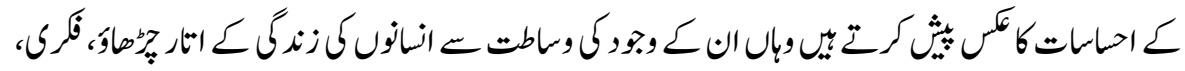

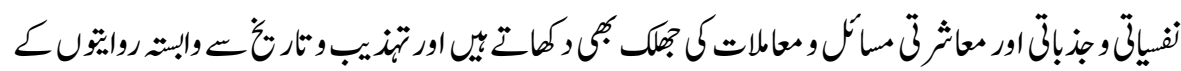
ائنبن جاجتيّ-

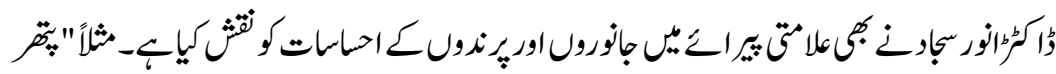

$$
\text { : }
$$

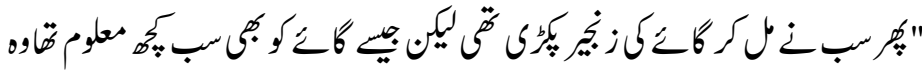

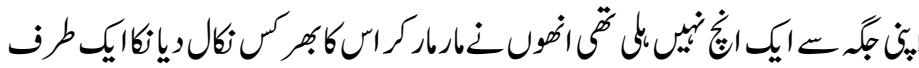

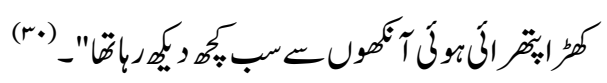

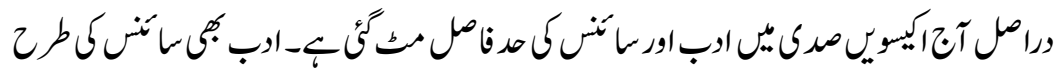

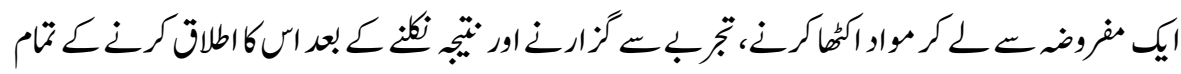

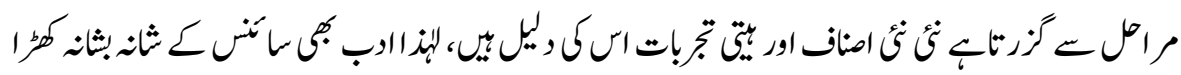

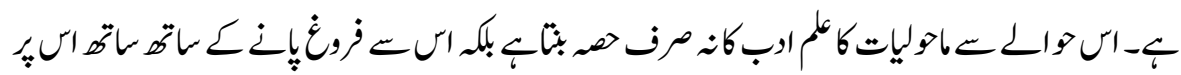


ISSN (Print): 2709-9636 | ISSN(Online) : 2709-9644 | ISSN-L : 2709-9636

家

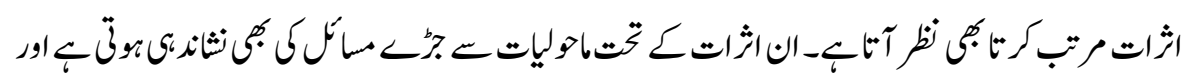

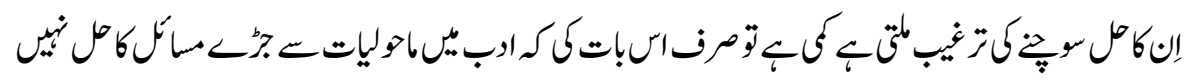

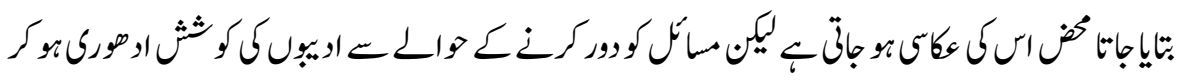

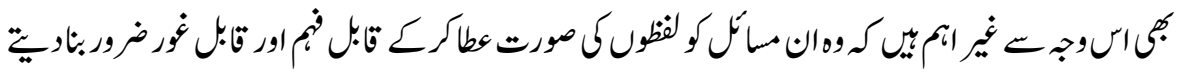

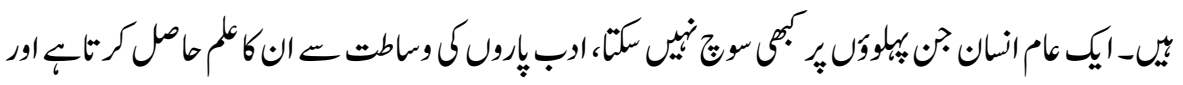

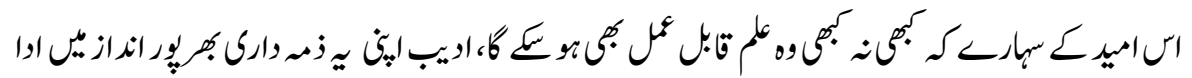

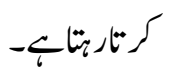

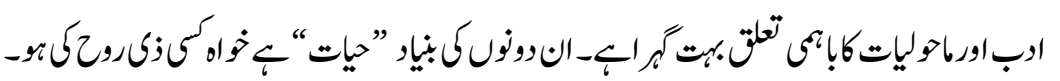

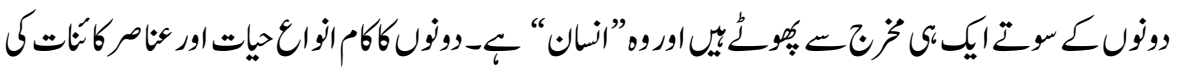

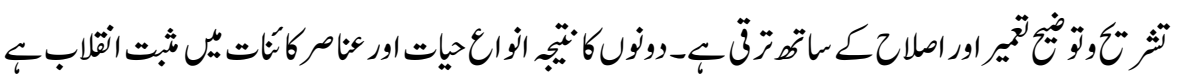

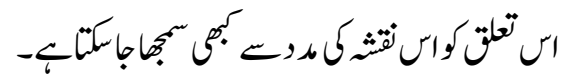

كاولياتهوم<smiles>C1=CCSCC2=CC(=C1)CSC2</smiles>

ارب

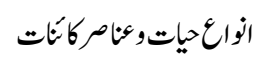

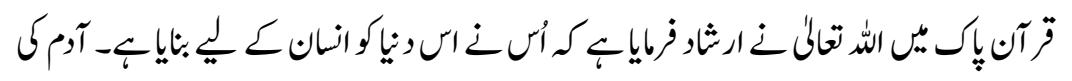

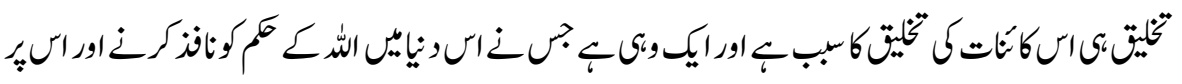

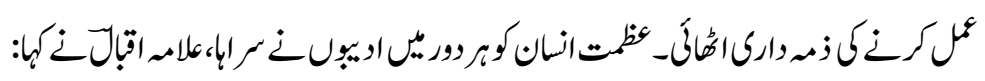

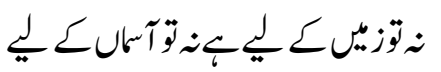
يجان

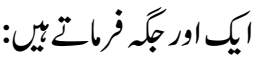

ar 


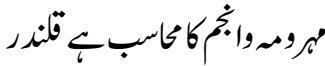

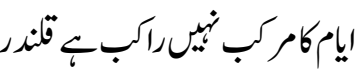

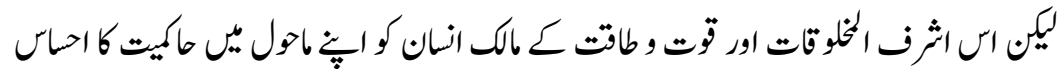

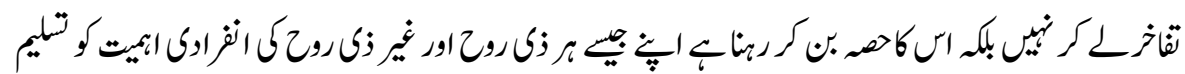

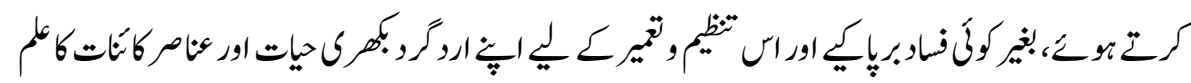

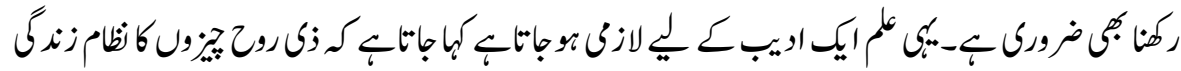

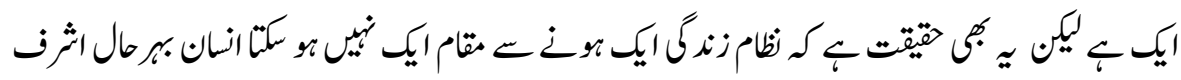

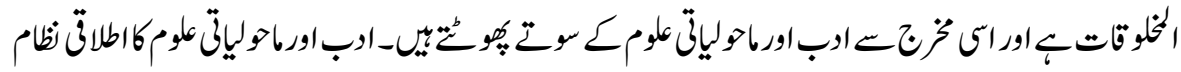

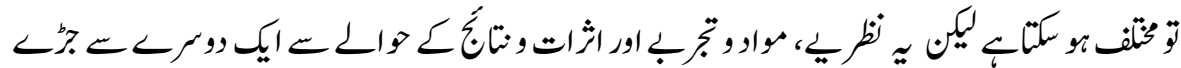
-

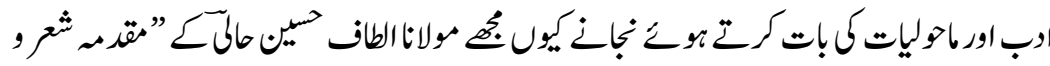

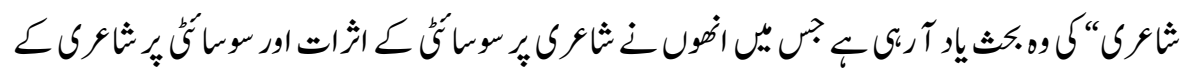

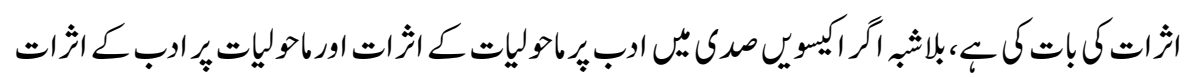

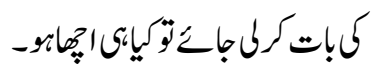

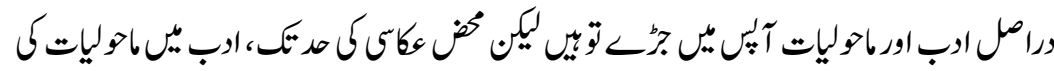

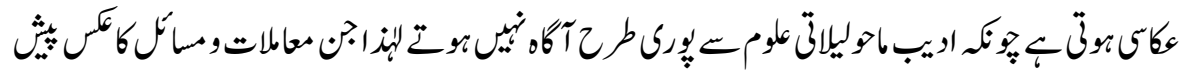

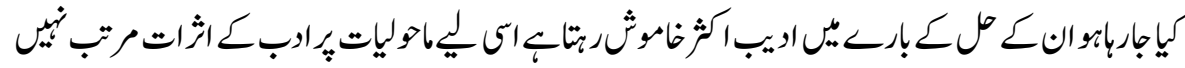

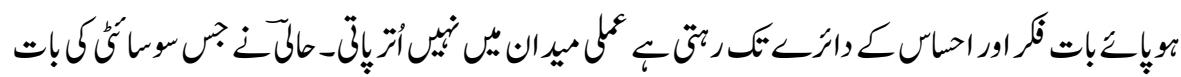

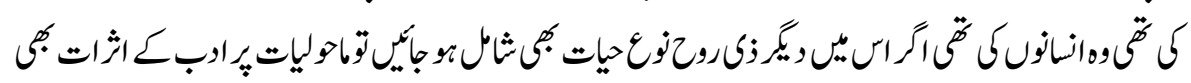

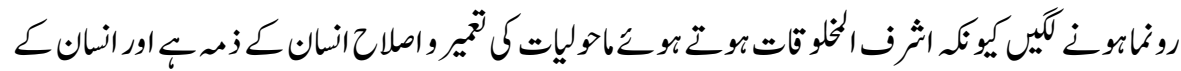

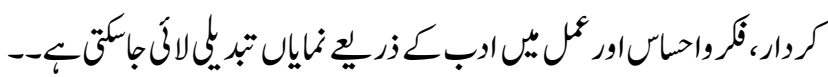

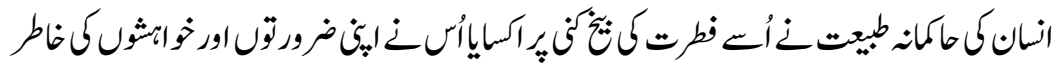

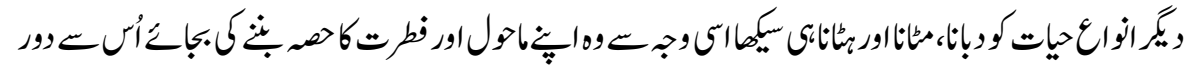




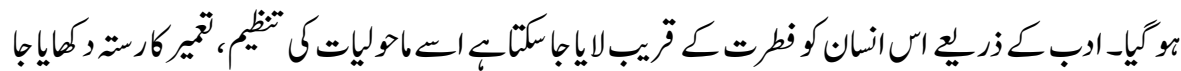

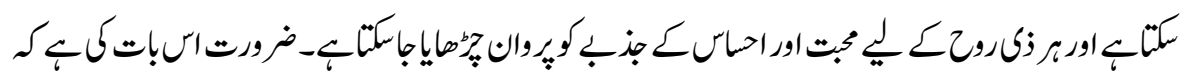

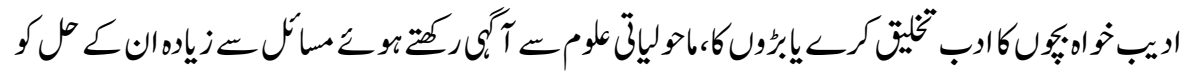

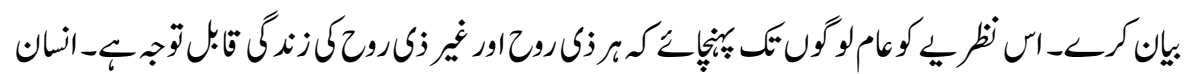

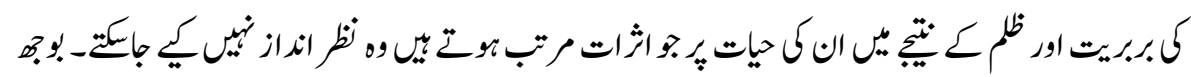

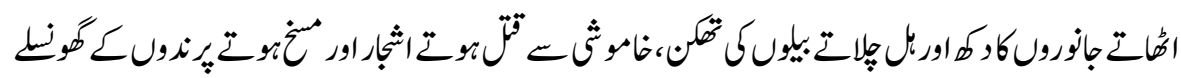

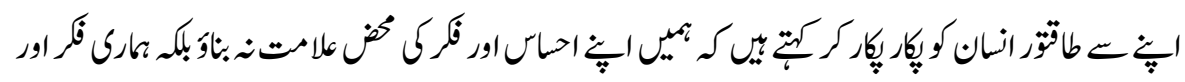
احساسكوم كرو

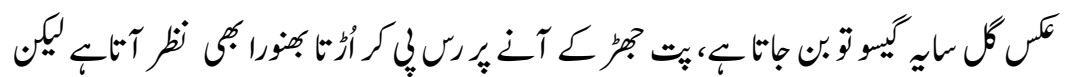

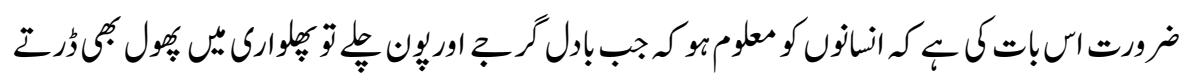
ب

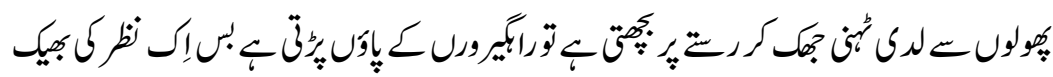

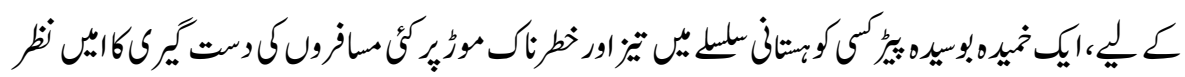

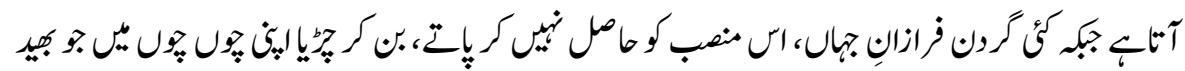

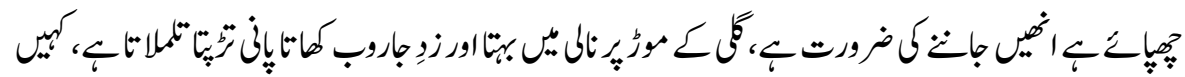

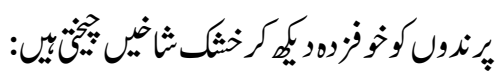

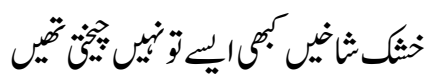

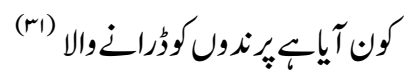

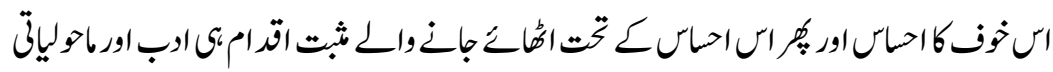

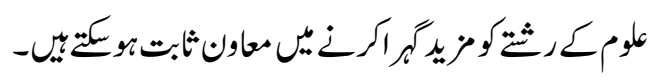

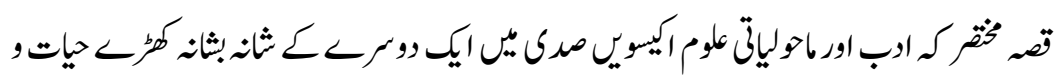

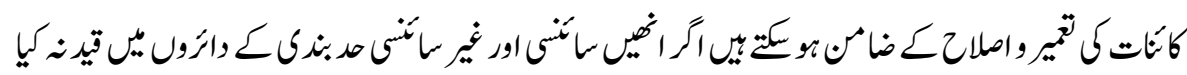

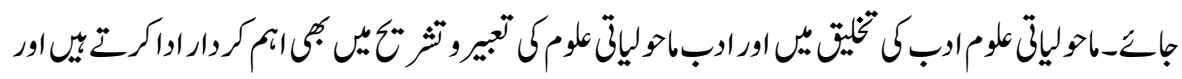




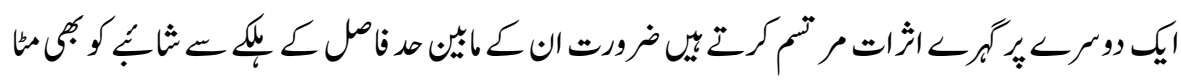

و توالـ جات

www.britanica.com $\quad .1$

Ibid $\quad r$

www.wikipedia.com $\quad . r$

Colin P. Towsend, Micheal Begon, Essentials of Ecology, (Second rr

Edition) Blackwell Publishing Company First Indian Reprint 2006,

$$
\text { Pg.4 }
$$

Edward J. Kormondy, Concepts of Ecology (Fourth Edition) PHI

Learning Private Limited, New Delhi: 2009, Pg. 3-4

Ibid, Pg. $4 \quad$. $\quad 4$

Ibid, Pg. $4 \quad$. .

A-Bala Subramanian, Introduction to Ecology, .^ www.researchgate.net/ publications/310021474-8.9.2005

Lyrical Bellads, Edited by RL Bvettand A.R Jones (Second Edition) $\quad .9$

Routhedge London and New York: 1991

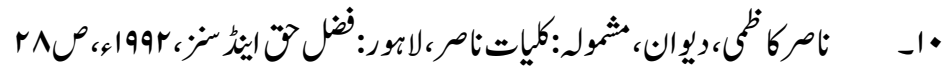

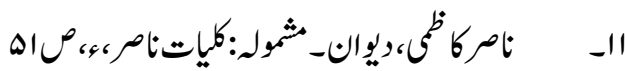

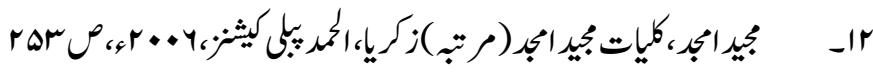

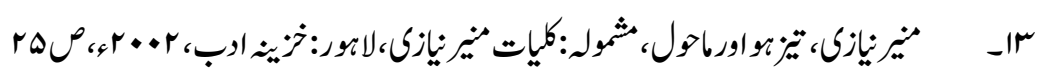

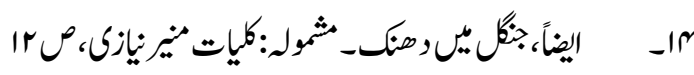

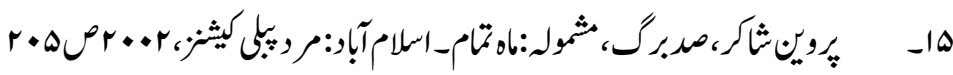




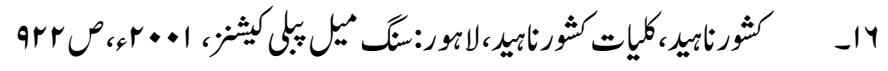

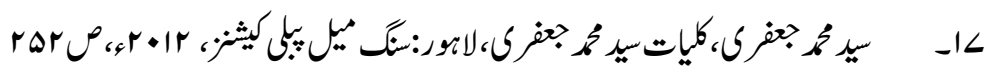

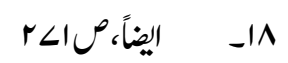

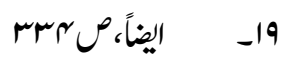

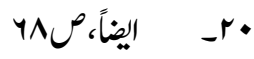

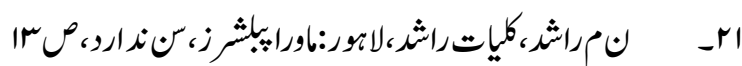

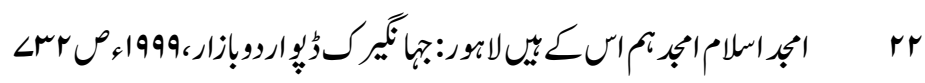

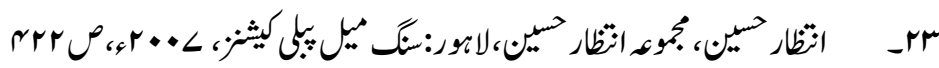
1

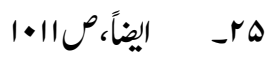
raLCص اليضاً

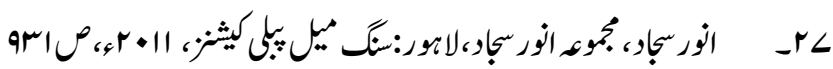
م اليضا، صاهـ

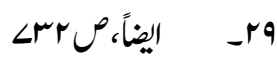

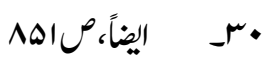

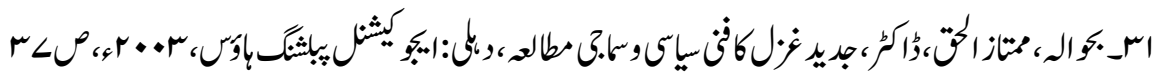

